\title{
Reduced miR-144-3p expression in serum and bone mediates osteoporosis pathogenesis by targeting RANK
}

\begin{tabular}{|c|c|}
\hline Journal: & Biochemistry and Cell Biology \\
\hline Manuscript ID & bcb-2017-0243.R2 \\
\hline Manuscript Type: & Article \\
\hline Date Submitted by the Author: & 20-Dec-2017 \\
\hline Complete List of Authors: & $\begin{array}{l}\text { Wang, Chunqing; Department of Orthopaedics, the Second Affiliated } \\
\text { Hospital of Soochow University } \\
\text { He, Hanliang; The Second Affiliated Hospital of Soochow University, } \\
\text { Department of Orthopaedics } \\
\text { Jiang, Yu; the Second Affiliated Hospital of Soochow University, } \\
\text { Department of Orthopaedics } \\
\text { Wang, Liang; the Second Affiliated Hospital of Soochow University, } \\
\text { Department of Orthopaedics } \\
\text { Xu, Youjia; The Second Affiliated Hospital of Soochow University }\end{array}$ \\
\hline $\begin{array}{r}\text { Is the invited manuscript for } \\
\text { consideration in a Special } \\
\text { Issue? : }\end{array}$ & $\mathrm{N} / \mathrm{A}$ \\
\hline Keyword: & osteoporosis, miR-144-3p, RANK, osteoclastogenesis \\
\hline
\end{tabular}


$1 \quad$ Reduced miR-144-3p expression in serum and bone mediates osteoporosis

2 pathogenesis by targeting RANK

3

4 Chunqing Wang ${ }^{1}$, Hanliang $\mathrm{He}^{1}$, Liang Wang ${ }^{1}$, Yu Jiang ${ }^{1}$, Youjia Xu

5

6

$7{ }^{1}$ Department of Orthopaedics, The Second Affiliated Hospital of Soochow University,

81055 Sanxiang Road, Suzhou 215004, China

9

10

11

12

13

14

15

16

17

18

Corresponding author: Youjia Xu

19

Email: xuyoujia@medmail.com.cn

20

Telephone: +86-0512-67783346

21

Fax: +86-0512-67783346

22

23 


\section{Abstract}

Osteoblasts and osteoclasts are responsible for the formation and resorption of bone, respectively. An imbalance between these two processes results in a disease called osteoporosis, in which a decreased level of bone strength increases the risk of a bone fracture. MicroRNAs (miRNAs) are small non-coding RNA molecules of 18-25 nucleotides that have been previously shown to control bone metabolism by regulating osteoblast and osteoclast differentiation. In the present study, we detected the expression pattern of 10 miRNAs in patient serum samples, and identified six miRNAs altered expression in patients with osteoporosis relative to non-osteoporosis.

We selected $m i R-144-3 p$ for further investigation, and showed that it regulates osteoclastogenesis by targeting $R A N K$ and that it is conserved amongst vertebrates. Disrupted expression of $m i R-144-3 p$ in CD14+ PBMCs changed TRAP activity and the osteoclast-specific genes TRAP, cathepsin $K$ (CTSK), and NFATC. TRAP staining, CCK-8 and flow cytometry analyses revealed that $m i R-144-3 p$ also affects osteoclast formation, proliferation and apoptosis. Together, these results indicate that miR-144-3p critically mediates bone homeostasis, and thus, represents a promising novel therapeutic candidate for the treatment of this disease.

Keywords: osteoporosis, $m i R-144-3 p, R A N K$, osteoclastogenesis

\section{Introduction}


The maintenance of bone homeostasis requires the correct balance between osteogenesis and bone resorption, as mediated by osteoblasts and osteoclasts, respectively (BENNETT et al. 2005) (MULARI et al. 2004). Imbalance between these two processes results in osteoporosis, which is characterized by a decrease in bone mineral density and by abNon-osteoporosis bone microarchitecture (BOLLERSLEV et al. 2005; LI et al. 2010). As life expectancy increases worldwide, a greater number of individuals suffer from osteoporosis; for example, almost half of the population in China is affected by the condition, and affected individuals suffer an average fracture ratio of almost 33\% (WANG et al. 2015; RouSE et al. 2016). Despite extensive research, the underlying mechanisms for this disease, and novel osteoporosis therapies, remain elusive (ADLER 2016).

Osteoclasts critically mediate bone building by enabling bone resorption (AMANO et al. 1998). The OPG/RANKL/RANK signalling pathway was recently identified as a classical pathway associated with osteoclast differentiation (WRIGHT et al. 2009).

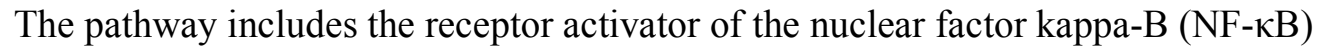
ligand (RANKL), which is an osteoclast differentiation factor and receptor of the receptor activator of NF- $\mathrm{KB}$ (RANK) (SOBACCHI et al. 2007). RANK is located in the cell membrane of osteoclasts, and functions to accelerate osteoclast activation and differentiation (HSU et al. 1999). Osteoprotegerin (OPG), also known as osteoclastogenesis inhibitory factor (OCIF) or tumour necrosis factor receptor superfamily member 11B (TNFRSF11B), is a decoy receptor that binds RANKL, and thereby prevents RANK-mediated NF- $\kappa$ B activation (BOYCE and XING 2007). A 
recent study successfully induced circulating CD14+ peripheral blood mononuclear cells (CD14+ PBMCs), which are early osteoclast progenitors, to differentiate into osteoclasts in vitro by activating either macrophage colony-stimulating factor (M-CSF) or RANKL (SHALHOUB et al. 2000; SORENSEN et al. 2007). This was possible because CD14+ PBMCs express RANK, which is activated by binding RANKL and subsequently induces osteoclast differentiation (Hsu et al. 1999).

MicroRNAs (miRNAs) are a class of small noncoding single stranded RNAs composed of 18-25 nucleotides (BARTEL 2004), which were first identified in $C$. elegans, but that have now been reported to play various crucial roles in cell development, proliferation, and differentiation (EBERT and SHARP 2012; MENDELL and OLSON 2012). Each miRNA regulates a given target gene by recognizing a 'seed sequence' site, (located in either the 3' untranslated region (3'UTR) or coding sequence (CDS) of the target gene), to promote degradation or inhibit translation of the target gene (RANA 2007; PAL et al. 2015). Recent research revealed that miRNA activity mediates bone homeostasis by regulating the expression of key osteoblast and osteoclast differentiation genes (VAN WIJNEN et al. 2013). For instance, miRNA-145 targets $S p 7$ to suppress osteogenic differentiation (JIA et al. 2013), $m i R$-21 has been reported to mediate mesenchymal stem cell (MSC) proliferation and differentiation during bone formation (ZHAO et al. 2015), and similarly, miR-214 targets FGFRI to attenuate osteogenic MSC differentiation (SHI et al. 2013). miRNAs have also been shown to affect osteoclast function, for example, overexpression and silencing of miR-503 in CD14+ PBMCs was recently found to inhibit and promote 
RANK-induced osteoclastogenesis, respectively, via the targeting of RANK (CHEN et al. 2014). In contrast, miRNAs are themselves targets of many regulators that affect bone differentiation. For instance, $D L X 3$ is a crucial regulator of hair follicle differentiation and cycling, but also targets $m i R-124$ in bone tissue to negatively regulate osteoclastic differentiation (ZHAO et al. 2016). Thus, miRNAs have been identified to regulate bone homeostatic processes, and in fact, the importance and complexity of this miRNA network suggests that continued research is required to elucidate the extent to which it affects osteoporosis pathogenesis.

In the clinical setting, diagnosis of osteoporosis was predominantly achieved by measuring bone mineral density and bone quality, where a loss of bone mineral density is indicative of osteoporosis. Early diagnosis is beneficial in facilitating an optimal treatment regime. Serum or systemically circulating miRNAs have been described in many diseases, including many types of cancer, as well as myocardial conditions, endometriosis, gastrointestinal disease, and/or diabetes mellitus (DEVAUX et al. 2015). Various miRNAs have been previously reported as serum biomarkers for osteoporosis; for example, Seeliger et al. reported miRNA-21, -23a, -24, -25, -100, and $-125 b$ to be expressed at a significantly higher level in the serum and bone tissues of patients with osteoporosis as compared to healthy individuals (SEELIGER et al. 2014). Very recently, Chen et al. described 15 serum miRNAs that were differentially expressed in OVX rats (an osteoporosis animal model), as identified and validated by microarray analysis and quantitative real-time PCR (qRT-PCR) (CHEN et al. 2016).

Taken together, these previous studies suggest that miRNAs are promising biomarkers 
112

113

114

115

116

117

118

119

for the early diagnosis of osteoporosis.

In the present study, we used qRT-PCR to reveal that $m i R-144-3 p$ is

downregulated in osteoporotic serum and bone tissues, and thus is a likely biomarker

for the diagnosis of osteoporosis. We also identified conserved $m i R-144-3 p$ target sites in the RANK 3'UTR via bioinformatics analysis, and demonstrated that overexpression of $m i R-144-3 p$ inhibited $R A N K$ expression in vitro to mediate osteoclast formation, proliferation, and apoptosis. Thus, we conclude that $m i R-144-3 p$ targets osteoclasts via $R A N K$. Our research provides a novel candidate biomarker for the diagnosis of osteoporosis in the clinical setting.

\section{Materials and Methods}

\section{Primary human samples}

Recruited patients admitted to our clinic with hip fractures were separated into two groups, comprising patients with osteoporosis who had a fracture (subject group), and patients non-osteoporosis but who had a fracture (control group). Blood and bone samples were collected during implantation of a total endoprosthesis or gamma nail into the proximal femur. The Local Ethical Review Committee of The Second Affiliated Hospital of Soochow University approved the present study.

\section{Sample processing, miRNA extraction, and qRT-PCR}

miRNAs from serum were extracted using the miRNeasy Serum/Plasma Kit

(Qiagen), according to the manufacturer's instructions. miRNA and mRNA were 
extracted from bone tissue using TRIzol, as per the manufacturer's instructions. miRNA was subsequently transcribed to generate cDNA using the PrimeScript ${ }^{\mathrm{TM}}$ One Step cDNA Synthesis Kit (Takara), and mRNA was transcribed to first strand cDNA using the First Strand cDNA Synthesis Kit (Takara) for gene expression analysis in bone tissue. qRT-PCR was performed using the ABI 7000 RT-PCR system (ABI, USA), with the listed primers (Table 1) and PrimeScript ${ }^{\mathrm{TM}}$ RT reagent kit (Takara, Japan), according to the manufacturer's instructions.

\section{CD14+ PBMC cultures}

Whole blood was obtained using a protocol approved by the Local Research Ethics Committee, and PBMCs were isolated as previously described (SORENSEN et al. 2007). CD14+ PBMCs were purified using CD14 antibody-coated magnetic cell sorting (MACS) MicroBeads (Miltenyi Biotec), and the purity of these cells was assessed using flow cytometry. When the CD14+ PBMCs purity was more than $90 \%$, subsequent experiments were carried out. CD14+ PBMCs (density $2.5 \times 10^{5}$ cells/well, in a 48 -well cluster plate) were cultured $\left(37^{\circ} \mathrm{C}\right.$, in a humidified atmosphere with $5 \% \mathrm{CO}_{2}$ ) in alpha minimum essential medium (a-MEM) supplemented with $10 \%$ foetal bovine serum (FBS), penicillin $(50 \mathrm{IU} / \mathrm{mL})$, and streptomycin $(50 \mathrm{mg} / \mathrm{mL})$. Osteoclastic differentiation was induced by replacing this medium with that supplemented with $25 \mathrm{ng} / \mathrm{mL}$ recombinant M-CSF and $25 \mathrm{ng} / \mathrm{mL}$ human RANKL (R\&D Systems, Minneapolis). 


\section{Luciferase activity assay}

A segment of the wild type (WT) RANK 3'UTR cloned into the pMir-reporter vector (Ambion), and a mutant $R A N K 3^{\prime} \mathrm{UTR}$ was generated by altering the predicted miR-144-3p RANK 3'UTR binding site via a two-step PCR approach. HEK 293T cells were co-transfected with either a WT or mutant $R A N K 3^{\prime}$ UTR reporter vector, and the miR-144-3p mimic or negative control constructs, cultured for $24 \mathrm{~h}$, and then assessed to ascertain their exhibited luciferase activity using a dual-luciferase reporter assay system (Promega).

\section{Tartrate-resistant acid phosphatase (TRAP) staining}

TRAP staining was performed using a commercially available TRAP staining kit according to the manufacturer's instructions (Sigma). Osteoclasts were identified as

TRAP-positive multinucleated (more than or equal to three nuclei) cells. The number of TRAP-positive multinucleated giant cells were counted in a 48-well culture plate using a Leica DM4000B microscope and photographed using a Leica DFC450c camera.

\section{Cell proliferation assay}

Cell proliferation was analysed using the Cell Counting Kit 8 (CCK-8; Dojindo).

After 6 days, CD14+ PBMCs were inducible, differently treated cells were then incubated for another $24 \mathrm{~h}$. The optical density (OD) of each group was measured at $450 \mathrm{~nm}$ using a BioTek microplate reader. 
Cell apoptosis assay

CD14+ PBMC apoptosis was analysed via the flow cytometry method (FCM), (PBS), and suspended in $500 \mu$ of binding buffer. They were then incubated $\left(37^{\circ} \mathrm{C}\right.$,

10 min) with Annexin V, stained with propidium iodide (PI), and analysed via the

FCM to determine their relative quantitative rate of apoptosis.

\section{Statistical analyses}

Data are presented as the mean $\pm \mathrm{SD}$. Statistical differences between two or more indicate statistical significance.

Results

qRT-PCR screening of differential miRNA expression in serum and bone samples of patients with and without osteoporosis

$$
\text { We selected } 10 \text { candidate miRNAs, which were reportedly related to bone }
$$
metabolism, to undergo qRT-PCR analysis to examine their differential expression in 
serum and bone samples of patients with and without osteoporosis (Fig. 1A). 15 non-osteoporosis and 45 osteoporosis sample was used. The non-osteoporosis sample we label with Non-osteoporosis and osteoporosis patient label with osteoporosis in the next experiments. All the sample clinic data was provided in the supplementary. The results of this analysis showed no difference in $m i R N A-7-5 p$ or $m i R-211-5 p$ expression, but a significant upregulation of miR-24-3p, 27a-3p,100, 125b, and $122 a$ expressions in both the serum and bone samples of osteoporosis with as compared to non-osteoporosis. The expression of miR-128 was found to be upregulated in bone tissue, but unchanged in the serum of osteoporosis with as compared to without non-osteoporosis. Conversely, miR-145 was upregulated only in the serum but not the bone tissue of osteoporosis. While $m i R-144-3 p$ has not been previously reported to be associated with osteoporosis, the results of the present study show that it was significantly downregulated in both the serum and bone samples of patients with as compared to non-osteoporosis (Fig. 1B, C). Thus, the generated data clearly demonstrate differential miRNA expression in osteoporosis, as compared to those not affected by the disease.

\section{miRNA-144-3p mediates gene expression in osteoporosis}

We selected $m i R-144-3 p$ as the focus for our subsequent analyses investigating the effects of miRNA activity on the pathogenesis of osteoporosis. We first used TargetScan online software (http://www.targetscan.org/vert-71/) to predict $m i R-144-3 p$ targets and refined the resultant list to include only those candidate genes 
222

223

224

225

226

227

228

229

230

231

232

233

234

235

236

237

238

239

240

241

242

243

associated with bone metabolism; the list comprised of BMPR1A, COL11A, SMAD4, $E S R R G$, and $R A N K$. To investigate the expression patterns of these candidate genes, total mRNA was extracted from patient samples and subjected to qRT-PCR. The results of this analysis showed that BMPR1A, COL11A, and ESRRG expression was unchanged between patients with and without osteoporosis (Fig 2A, B, D); however, the expression of SMAD4 and $R A N K$ was significantly upregulated in patients with osteoporosis as compared to those without the disease (Fig 1C, E). A previous report demonstrated that $m i R-144-3 p$ regulates osteogenic differentiation, as well as the proliferation of murine MSCs by specifically targeting Smad4; thus, we selected $R A N K$ to be the focus of our subsequent analyses. We used ELISA to examine the RANK content in patient serum samples, and resultantly showed that the concentration of RANK was significantly higher in patients with osteoporosis than in those without osteoporosis (Fig $1 \mathrm{~F})$. This suggests that $R A N K$ concentration is regulated by $m i R-144-3 p$.

\section{$R A N K$ is a direct $m i R-144-3 p$ target gene}

TargetScan online software was used to identify a probable single miR-144-3p target site in the $R A N K 3^{\prime} \mathrm{UTR}$ that was comprised of nine nucleotides homologous with the miR-144-3p consensus seed sequence. Furthermore, this $R A N K 3^{\prime} \mathrm{UTR}$ miR-144-3p target site was found to be conserved in humans, chimps, mice, dogs, and lizards (Fig 3A).

$$
\text { To investigate the potential differential expression of } m i R-144-3 p \text { during }
$$


osteoclastogenesis, CD14+ PBMCs from patients unaffected by osteoporosis were induced to differentiate via M-CSF and RANKL treatment. These cells were cultured for 6 days, and then analysed via qRT-PCR to determine their miR-144-3p expression. The results of this analysis showed that the CD14+ PBMC miR-144-3p expression was significantly downregulated in response to the induction of differentiation as compared to undifferentiated cells (at 0 days) (Fig 3A). The miR-144-3p negative control, inhibitor, and mimic were next transfected into CD14+ PBMCs, which were subsequently induced to differentiate via treatment with M-CSF and RANKL, and collected after 6 days of culture. qRT-PCR analysis of $R A N K$ expression at this point revealed it to be increased and decreased in the inhibitor- and mimic-transfected cells, respectively, compared with the negative control-transfected cells (Fig 3C). These data suggest that $R A N K$ is a $m i R-144-3 p$ target in CD14+ PBMCs.

To validate this hypothesis, we cloned a WT and mutant (seed sequence) $R A N K$ 3'UTR into the pMir-reporter vector to generate a CMV-luciferase-WT and CMV-luciferase-mutant RANK 3'UTR expression vector, respectively (Fig 3D). Dual-luciferase reporter assays were then conducted by co-transfecting HEK 293T cells with these expression vectors and the $m i R-144-3 p$ negative control, mimic, and/or inhibitor. The results of these analyses showed that luciferase activity was significantly decreased in response to co-transfection of the CMV-luciferase-WT $R A N K$ 3'UTR and $m i R-144-3 p$ mimic, as compared to co-transfection of the CMV-luciferase-WT RANK 3'UTR and the miR-144-3p negative control. In contrast, co-transfection of the CMV-luciferase-mutant RANK 3'UTR and miR-144-3p mimic 
failed to inhibit luciferase activity (Fig 3E). Similarly, western blot analysis of RANK protein levels in CD14+ PBMCs transfected with the miR-144-3p negative control, inhibitor, and/or mimic showed RANK production to be increased and decreased in response to transfection of the $m i R-144-3 p$ inhibitor and mimic, respectively, as compared to RANK levels in the negative control (Fig 3F). Together, these data confirm $R A N K$ as a $m i R N A-144-3 p$ target.

miR-144-3p regulates the osteoclastogenesis of CD14+ PBMCs following M-CSF and RANKL treatment

To investigate the role of $m i R N A-144-3 p$ during osteoclastogenesis, CD14+ PBMCs from a patient unaffected by osteoporosis were transfected with the miR-144-3p negative control, mimic, and/or inhibitor, treated with M-CSF and RANKL, cultured for 6 days, and then analysed to evaluate their expression of the osteoclast-specific genes TRAP, cathepsin K (CTSK), and NFATC, and for their exhibited TRAP activity. The results of these analyses showed that treating the CD14+ PBMCs with the miR-144-3p mimic and inhibitor significantly decreased and increased TRAP activity, respectively, as compared with the negative control (Fig 4A). Similarly, the qRT-PCR analysis demonstrated that TRAP, CTSK, and NFATC mRNA expression was down- and up-regulated in response to treating the CD14+ PBMCs with the miR-144-3p mimic and inhibitor, respectively, as compared to treating them with the negative control (Fig 4B-D). These results suggest that $m i R-144-3 p$ negatively regulates osteoclastogenesis. 
miR-144-3p affects osteoclast formation following the M-CSF and RANKL treatment of CD14+ PBMCs

To explore the role of $m i R-144-3 p$ in osteoclast formation, TRAP staining experiments were performed. The results showed that the number of TRAP-positive cells decreased in the $m i R-144-3 p$ mimic group, and the number of TRAP-positive cells increased in the $m i R-144-3 p$ inhibitor group as compared with the negative control group (Fig. 5A B). These results demonstrated that miR-144-3p affects osteoclast formation.

\section{miR-144-3p mediates the proliferation and apoptosis of M-CSF/RANKL-induced}

\section{osteoclasts}

To investigate the role of $m i R-144-3 p$ in cell proliferation and apoptosis during osteoclastogenesis, CD14+ PBMCs were transfected with the miRNA-144-3p negative control, inhibitor, and/or mimic, and induced to differentiate via treatment with M-CSF and RANKL. These cells were cultured for 6 days, and then subjected to

CCK-8 assay and flow cytometric analysis (Fig 5A). The results of the CCK-8 assay revealed that treatment with the $m i R-144-3 p$ mimic and inhibitor reduced and increased CD14+ PBMC proliferation, respectively, as compared to treatment with the negative control (Fig 5B). Similarly, the results of the flow cytometric analysis showed that transfection of the $m i R-144-3 p$ mimic increased CD14+ PBMC apoptosis as compared to transfection with the negative control; however, no difference was 
310

311

312

313

314

315

316

317

318

319

320

321

322

323

324

325

326

327

328

329

330

331

observed in response to transfecting CD14+ PBMCs with the miR-144-3p inhibitor

(Fig 5C). Together, these data indicate that miR-144-3p mediates apoptosis and cell proliferation during osteoclastogenesis.

\title{
Discussion
}

Osteoporosis is a disorder characterised by a loss of balance between osteoblast and osteoclast activities (LI et al. 2010). An increasing proportion of aging individuals worldwide are affected by this condition; thus, study into novel therapeutic interventions is urgently required to mitigate the increasing medical and socioeconomic burden caused by osteoporosis (WANG et al. 2015). miRNAs critically mediate osteoporosis pathogenesis, and many previous studies have revealed that this occurs via their exerted effects on both osteoblasts and osteoclasts (VAN WIJNEN et al. 2013). In the present study, we identified $m i R-144-3 p$ as an osteoporosis-associated miRNA that targets $R A N K$ to mediate osteoclastogenesis, and whose differential expression likely contributes to the pathogenesis of this disease.

\section{miR-144-3p is a novel serum biomarker for osteoporosis}

\author{
Extensive research into the use of serum-based miRNAs as disease biomarkers
} has been conducted over the last several years (DEVAUX et al. 2015; RouSE et al. 2016). Furthermore, various osteoporosis miRNA biomarkers have been investigated as potential diagnostic tools in the clinical setting (MENG et al. 2015). For example, in 2014, Seeliger et al. reported five freely circulating serum and bone tissue-contained 
332

333

334

335

336

miRNAs (miR-21, 23a, 24, 25, 100, and 125b) that were associated with osteoporotic fractures (SEELIGER et al. 2014), and more recently, Chen et al. identified 15 serum miRNA osteoporosis biomarkers (CHEN et al. 2016). Meng et al. and Zat et al. identified $m i R-194-5 p$ and $m i R-422 a$, respectively, as potential biomarkers for postmenopausal osteoporosis (CAO et al. 2014; MENG et al. 2015), and similarly, Wang et al. reported $m i R-133 a$ in human circulating monocytes as an additional candidate biomarker associated with postmenopausal osteoporosis (WANG et al. 2012).

In the present study, we selected and assessed the expression of 10 miRNAs in patients with osteoporosis, two of which ( $m i R-100$ and $m i R-125 b)$ were previously evaluated by Seeliger et al. (SEELIGER et al. 2014). The results of the present study are consistent with those of Seeliger et al., which showed that both miR-100 and $m i R-125 b$ were upregulated in patients with osteoporosis. $m i R-122 a$ was also found in the present study to be upregulated in both the serum and bone samples of patients with, as compared to Non-osteoporosis. Conversely, miR-128 and miR-145a were shown to be elevated only in the bone and serum samples, respectively, of patients with osteoporosis. Of these, $m i R-145 a$ has been previously shown to suppress osteogenic differentiation by targeting $S p 7$ (JIA et al. 2013). In the present study, we found $m i R-144-3 p$ to be differentially expressed in osteoporotic serum and bone samples, suggesting that it is a promising novel biomarker for the diagnosis of osteoporosis in the clinical setting. Although many miRNAs have, to date, been identified as candidate osteoporosis biomarkers, their effect on osteoporosis 
354

355

356

357

358

359

360

361

362

363

364

365

366

367

368

369

370

371

372

373

374

375

pathogenesis is not well understood。

\section{$R A N K$ is a direct target of $m i R N A-144-3 p$}

miRNAs regulate gene expression by recognising the 3'UTR or CDS of various

target genes (BARTEL 2004). Of these target genes, SMAD4 and RANK were

established to exhibit differential expression in patients with osteoporosis via

qRT-PCR. We selected $R A N K$ for further analysis, since $S M A D 4$ has been previously

reported to be targeted by $m i R-144-3 p$ during its regulation of murine MSC

osteogenic differentiation and proliferation (HUANG et al. 2016). RANK exists in the

cell membrane, and after binding to its ligand, mediates osteoclast differentiation. Our

loss-of-function and overexpression experiments revealed that RANK is a target gene for $m i R-144-3 p$.

miR-144-3p targets $R A N K$ to regulate osteoclastogenesis

$m i R-144-3 p$ regulates osteoclastogenesis by targeting $R A N K$. This was shown in the present study with both loss-of-function and overexpression experiments for miR-144-3p in vitro. As a known type II membrane protein and a member of the tumour necrosis factor (TNF) superfamily (BOYCE and XING 2007; WrigHT et al. 2009), RANKL induces osteoclast differentiation by binding and activating RANK in myeloid-lineage cells (BOYCE and XING 2007; BOYCE and XING 2008). RANK may also bind to OPG, which is a protein secreted predominantly by cells of the osteoblast lineage that potently inhibits osteoclast formation by preventing the binding of RANKL to RANK (WRIGHT et al. 2009). In the present study, we revealed that miR-144-3p inhibits TRAP activity. It is well established that the bone matrix 
phosphoproteins osteopontin and bone sialoprotein are highly efficient in vitro TRAP substrates that bind to osteoclasts when phosphorylated (HAYMAN 2008). Furthermore, TRAP activity has been previously shown to be an osteoclast marker (HALLEEN et al. 2006; Wu et al. 2009), and increased TRAP activity is considered to be indicative of a high rate of osteoclast differentiation. In the present study, we also examined the expression of two key genes, NFATC1 and CTSK, in PBMCs after the transfection with the miR-144-3p mimic and inhibitor. NFATC1 is involved in the NFATC1 signalling pathway, which is critical to the regulation of osteoclast differentiation and maturation (CROTTI et al. 2006; KIM and KIM 2014). CTSK has been shown to be predominantly involved in degrading the organic bone matrix and controlling osteoclast population size by regulating apoptosis (HENRIKSEN et al. 2006; FULLER et al. 2008). In the present study, transfection of the miR-144-3p mimic and inhibitor induced a significant decrease and increase, respectively, in the expression of both NFATC1 and CTSK. Furthermore, transfection of both constructs affected cell proliferation and apoptosis, strongly suggesting that $m i R-144-3 p$ affects osteoclastogenesis by targeting $R A N K$.

The results of the present study also identified SMAD4 as being differentially expressed in response to modulating $m i R N A-144-3 p$ expression. SMAD4 is known to be activated by BMP signalling and to predominantly mediate osteoblastogenesis. In fact, a recent study showed that $m i R N A-144-3 p$ could target SMAD4 to regulate osteoblastogenesis (HUANG et al. 2016); thus, $m i R-144-3 p$ appears to not only participate in osteoclastogenesis, but also osteoblastogenesis. 
398

399

400

401

402

403

404

405

406

407

408

409

410

411

412

413

414

415

416

417

418

419

420

421

422

423

424

425

426

427

In summary, we conclude from the results of the present study that miR-144-3p

should be considered as a serum osteoporosis biomarker for use in the clinical setting. miR-144-3p via direct targeting of $R A N K$ mediates osteoclastogenesis. Thus, miRNA-144-3p may be a promising novel therapeutic candidate for the treatment osteoporosis.

\section{Acknowledgments}

This work was supported by grants from the National Natural Science Foundation of China (81572179), and the Science and Technology Foundation of Guiyang (20161001-46).

\section{Conflict of interest}

All authors have no conflicts of interest.

\section{References}

Adler, R. A., 2016 Osteoporosis treatment: complexities and challenges. J Endocrinol Invest 39: 719-720.

Amano, H., S. Yamada and R. Felix, 1998 Colony-stimulating factor-1 stimulates the fusion process in osteoclasts. J Bone Miner Res 13: 846-853.

Bartel, D. P., 2004 MicroRNAs: genomics, biogenesis, mechanism, and function. Cell 116: 281-297.

Bennett, C. N., K. A. Longo, W. S. Wright, L. J. Suva, T. F. Lane et al., 2005 Regulation of osteoblastogenesis and bone mass by Wnt10b. Proc Natl Acad Sci U S A 102: 3324-3329.

Bollerslev, J., S. G. Wilson, I. M. Dick, F. M. Islam, T. Ueland et al., 2005 LRP5 gene polymorphisms predict bone mass and incident fractures in elderly Australian women. Bone 36: 599-606.

Boyce, B. F., and L. Xing, 2007 Biology of RANK, RANKL, and osteoprotegerin. Arthritis Res Ther 9 Suppl 1: S1.

Boyce, B. F., and L. Xing, 2008 Functions of RANKL/RANK/OPG in bone modeling and remodeling. Arch Biochem Biophys 473: 139-146.

Cao, Z., B. T. Moore, Y. Wang, X. H. Peng, J. M. Lappe et al., 2014 MiR-422a as a potential cellular microRNA biomarker for postmenopausal osteoporosis. PLoS One 9: e97098.

Chen, C., P. Cheng, H. Xie, H. D. Zhou, X. P. Wu et al., 2014 MiR-503 regulates osteoclastogenesis via 
targeting RANK. J Bone Miner Res 29: 338-347.

Chen, J., K. Li, Q. Pang, C. Yang, H. Zhang et al., 2016 Identification of suitable reference gene and biomarkers of serum miRNAs for osteoporosis. Sci Rep 6: 36347.

Crotti, T. N., M. Flannery, N. C. Walsh, J. D. Fleming, S. R. Goldring et al., 2006 NFATc1 regulation of the human beta3 integrin promoter in osteoclast differentiation. Gene 372: 92-102.

Devaux, Y., M. Mueller, P. Haaf, E. Goretti, R. Twerenbold et al., 2015 Diagnostic and prognostic value of circulating microRNAs in patients with acute chest pain. J Intern Med 277: 260-271.

Ebert, M. S., and P. A. Sharp, 2012 Roles for microRNAs in conferring robustness to biological processes. Cell 149: 515-524.

Fuller, K., K. M. Lawrence, J. L. Ross, U. B. Grabowska, M. Shiroo et al., 2008 Cathepsin K inhibitors prevent matrix-derived growth factor degradation by human osteoclasts. Bone 42: 200-211.

Halleen, J. M., S. L. Tiitinen, H. Ylipahkala, K. M. Fagerlund and H. K. Vaananen, 2006 Tartrate-resistant acid phosphatase 5b (TRACP 5b) as a marker of bone resorption. Clin Lab 52: 499-509.

Hayman, A. R., 2008 Tartrate-resistant acid phosphatase (TRAP) and the osteoclast/immune cell dichotomy. Autoimmunity 41: 218-223.

Henriksen, K., M. G. Sorensen, R. H. Nielsen, J. Gram, S. Schaller et al., 2006 Degradation of the organic phase of bone by osteoclasts: a secondary role for lysosomal acidification. J Bone Miner Res 21: 58-66.

Hsu, H., D. L. Lacey, C. R. Dunstan, I. Solovyev, A. Colombero et al., 1999 Tumor necrosis factor receptor family member RANK mediates osteoclast differentiation and activation induced by osteoprotegerin ligand. Proc Natl Acad Sci U S A 96: 3540-3545.

Huang, C., J. Geng, X. Wei, R. Zhang and S. Jiang, 2016 MiR-144-3p regulates osteogenic differentiation and proliferation of murine mesenchymal stem cells by specifically targeting Smad4. FEBS Lett 590: 795-807.

Jia, J., Q. Tian, S. Ling, Y. Liu, S. Yang et al., 2013 miR-145 suppresses osteogenic differentiation by targeting Sp7. FEBS Lett 587: 3027-3031.

Kim, J. H., and N. Kim, 2014 Regulation of NFATc1 in Osteoclast Differentiation. J Bone Metab 21: 233-241.

Li, W. F., S. X. Hou, B. Yu, M. M. Li, C. Ferec et al., 2010 Genetics of osteoporosis: accelerating pace in gene identification and validation. Hum Genet 127: 249-285.

Mendell, J. T., and E. N. Olson, 2012 MicroRNAs in stress signaling and human disease. Cell 148: 1172-1187.

Meng, J., D. Zhang, N. Pan, N. Sun, Q. Wang et al., 2015 Identification of miR-194-5p as a potential biomarker for postmenopausal osteoporosis. PeerJ 3: e971.

Mulari, M. T., Q. Qu, P. L. Harkonen and H. K. Vaananen, 2004 Osteoblast-like cells complete osteoclastic bone resorption and form new mineralized bone matrix in vitro. Calcif Tissue Int 75: 253-261.

Pal, M. K., S. P. Jaiswar, V. N. Dwivedi, A. K. Tripathi, A. Dwivedi et al., 2015 MicroRNA: a new and promising potential biomarker for diagnosis and prognosis of ovarian cancer. Cancer Biol Med 12: 328-341.

Rana, T. M., 2007 Illuminating the silence: understanding the structure and function of small RNAs. Nat Rev Mol Cell Biol 8: 23-36.

Rouse, R., B. Rosenzweig, K. Shea, A. Knapton, S. Stewart et al., 2016 MicroRNA biomarkers of pancreatic injury in a canine model. Exp Toxicol Pathol. 
Seeliger, C., K. Karpinski, A. T. Haug, H. Vester, A. Schmitt et al., 2014 Five freely circulating miRNAs and bone tissue miRNAs are associated with osteoporotic fractures. J Bone Miner Res 29: 1718-1728.

Shalhoub, V., G. Elliott, L. Chiu, R. Manoukian, M. Kelley et al., 2000 Characterization of osteoclast precursors in human blood. Br J Haematol 111: 501-512.

Shi, K., J. Lu, Y. Zhao, L. Wang, J. Li et al., 2013 MicroRNA-214 suppresses osteogenic differentiation of C2C12 myoblast cells by targeting Osterix. Bone 55: 487-494.

Sobacchi, C., A. Frattini, M. M. Guerrini, M. Abinun, A. Pangrazio et al., 2007 Osteoclast-poor human osteopetrosis due to mutations in the gene encoding RANKL. Nat Genet 39: 960-962.

Sorensen, M. G., K. Henriksen, S. Schaller, D. B. Henriksen, F. C. Nielsen et al., 2007 Characterization of osteoclasts derived from CD14+ monocytes isolated from peripheral blood. J Bone Miner Metab 25: 36-45.

van Wijnen, A. J., J. van de Peppel, J. P. van Leeuwen, J. B. Lian, G. S. Stein et al., 2013 MicroRNA functions in osteogenesis and dysfunctions in osteoporosis. Curr Osteoporos Rep 11: 72-82.

Wang, O., Y. Hu, S. Gong, Q. Xue, Z. Deng et al., 2015 A survey of outcomes and management of patients post fragility fractures in China. Osteoporos Int 26: 2631-2640.

Wang, Y., L. Li, B. T. Moore, X. H. Peng, X. Fang et al., 2012 MiR-133a in human circulating monocytes: a potential biomarker associated with postmenopausal osteoporosis. PLoS One 7: e34641.

Wright, H. L., H. S. McCarthy, J. Middleton and M. J. Marshall, 2009 RANK, RANKL and osteoprotegerin in bone biology and disease. Curr Rev Musculoskelet Med 2: 56-64.

Wu, Y., J. W. Lee, L. Uy, B. Abosaleem, H. Gunn et al., 2009 Tartrate-resistant acid phosphatase (TRACP $5 b)$ : a biomarker of bone resorption rate in support of drug development: modification, validation and application of the BoneTRAP kit assay. J Pharm Biomed Anal 49: 1203-1212.

Zhao, N., D. Han, Y. Liu, Y. Li, L. Zeng et al., 2016 DLX3 negatively regulates osteoclastic differentiation through microRNA-124. Exp Cell Res 341: 166-176.

Zhao, W., Y. Dong, C. Wu, Y. Ma, Y. Jin et al., 2015 MiR-21 overexpression improves osteoporosis by targeting RECK. Mol Cell Biochem 405: 125-133. 
Tables

501 Table 1 List of primers used in this study

\begin{tabular}{|l|l|l|l|}
\hline Gene & Forward primer & Reverse primer & Note \\
\hline nMPR1A & GCTTCATGGCACTGGGATG & CATCATCTGGACAGTGCCCT & qRT-PCR \\
\hline COL11A & CACCCTCATTGTTGACTGCA & GGCAGCAACCTCAAAGACTT & qRT-PCR \\
\hline SMAD4 & TGATCTATGCCCGTCTCTGG & CCAGGTGATACAACTCGTTCG & qRT-PCR \\
\hline ESRRG & GAATGGCCATCAGAACGGAC & CACACTTGGTCTGGGGATCT & qRT-PCR \\
\hline RANK & ACTACACCAAGTACCTGCGT & ACGAACATGGAGCGGGAG & qRT-PCR \\
\hline TRAP & ACCAATTCTGTGTCCTCGGA & TAATCGAGTGCAGGGGTTCC & qRT-PCR \\
\hline R $F A N K=$ & TTCCTGGATGTTTGGAAAC & GAGACATGAAGGTGAAGTAC & 3'UTR \\
\hline & TGCAGAAGAACCGGGGTATT & AGGGCTTTCTCATTCCCCTC & qRT-PCR \\
\hline
\end{tabular}

502

503 
504

505

506

507

508

509

510

511

512

513

514

515

516

517

518

519

520

521

522

523

524

525

Figure legends

Fig. 1 miRNA expression patterns in bone and serum samples from Non-osteoporosis and osteoporosis (A) Selected candidate miRNAs, and corresponding qRT-PCR primer sequences. (B) Expression patterns of the selected candidate miRNAs in patient serum samples. (C) Expression patterns of the selected candidate miRNAs in patient bone samples. Expression levels are represented as the mean \pm S.D, with statistical significance being assessed via the Student's $t$-test $(\mathrm{p}<0.05)$

\section{Fig. 2 Potential $m i R-144-3 p$ target genes expression patterns in non-osteoporosis} and osteoporosis. Online software was used to predict $m i R-144-3 p$ target genes, and the resultant list of candidates was refined to include only those genes associated with bone homeostasis. Selected candidate genes were verified to be differentially expressed between non-osteoporosis and osteoporosis, via qRT-PCR. (A-E) The expression patterns of (A) BMPR1A, (B) COL11A, (C) SMAD4, (D) ESRRG, and (E) RANK in non-osteoporosis and osteoporosis in bone tissue. (F) The level of RANK production in patient serum samples was assessed via ELISA. Expression levels are represented as the mean \pm S.D., with statistical significance being assessed via the Student's $t$-test $(\mathrm{p}<0.05)$.

Fig. $3 \boldsymbol{R A N K}$ is a miR-144-3p target gene (A) Using TargetScans online software, we identified a $m i R-144-3 p$ target site (red colour) in the RANK $3^{\prime}$ UTR that is 
526

527

528

529

530

531

532

533

534

535

536

537

538

539

540

541

542

543

544

545

546

547

conserved in humans, chimps, mice, dogs, and lizards. (B) The expression of miR-144-3p was measured in CD14+ PBMCs using qRT-PCR, six days after the cells were induced to differentiate. (C) CD14+ PBMCs were transfected with the $m i R-44-3 p$ mimic or inhibitor, and their expression of $R A N K L$ was then assessed via qRT-PCR. (D) A WT and mutant RANKL 3'UTR was cloned into the pMir-reporter vector to generate the CMV-luciferase- $R A N K L$ and CMV-luciferase- $R A N K L$ mutant 3'UTR vectors. (E) A cell-transfection experiment was performed to demonstrate that miR-144-3p inhibits RANKL expression in vivo. +, added; -, not added. (F) Western blot analysis demonstrating that $m i R-144-3 p$ affects RANKL protein levels in vivo. +, added; -, not added. Data represent the mean \pm S.D., with statistical significance being assessed by the Student's $t$-test $(\mathrm{p}<0.05)$.

\section{Fig. 4 miR-144-3p regulates the induction of CD14+ PBMCs to undergo} osteoclastogenesis via M-CSF and RANKL treatment (A) CD14+ PBMCs were induced to differentiate via M-CSF and RANKL treatment and cultured for six days, before they exhibited TRAP activity. Transfection of the miR-144-3p mimic and inhibitor was shown to decrease and increase CD14+ PBMC TRAP activity, respectively. (B-D) qRT-PCR analysis of (B) TRAP, (C) NFATC, and (D) CTSK mRNA expression in response to transfection of CD14+ PBMCs with the miRNA-144-3p negative control, mimic, or inhibitor. Data represent the mean \pm S.D., with statistical significance being assessed by the Student's t-test $(\mathrm{p}<0.05)$. 
548

549

550

551

552

553

554

555

556

557

558

559

560

561

Fig. 5 miR-144-3p regulates osteoclast formation following the M-CSF and RANKL treatment of CD14+ PBMCs. (A) The cells were induced by the addition of M-CSF+RANKL for 6 days, after which the miR-144-3p mimic and inhibitor was added. (B) The statistics of the number of TRAP-positive cells. Statistical significance was assessed using the Student's $t$-test $(\mathrm{p}<0.05)$.

Fig. 6 Effects of transfecting CD14+ PBMCs with the miR-144-3p mimic, inhibitor, or negative control on cell proliferation and apoptosis (A) A CCK-8 assay was performed to evaluate the effect of transfecting CD14+ PBMCs with the miR-144-3p mimic, inhibitor, and/or negative control on cell proliferation. (B) A statistical analysis of the results revealed that the miR-144-3p mimic and inhibitor inhibited and increased CD14+ PBMC proliferation, respectively. (C) The effects of miR-144-3p mimic, inhibitor, and/or negative control transfection on CD14+ PBMC apoptosis was analysed via flow cytometry. Data represent the mean \pm S.D., with statistical significance being assessed by the Student's $t$-test $(\mathrm{p}<0.05)$. 
A

\begin{tabular}{ll}
\hline \multicolumn{1}{c}{ Name } & \multicolumn{1}{c}{ Sequence } \\
\hline hsa-miR-7-5p & Forward: 5'-GGAAGACTAGTGATTTTGTTG -3' \\
hsa-miR-24-3p & Forward: 5'-GGCTCAGTTCAGCAGGAACAG -3' \\
hsa-miR-27a-3p & Forward: 5'-CCTTCACAGTGGCTAAGTTCC-3' \\
hsa-miR-100 & Forward: 5'-CCCGTAGATCCGAACTTGTG-3' \\
hsa-miR-125b & Forward: 5'-TCCCTGAGACCCTAACTTGTG-3' \\
hsa-miR-128 & Forward: 5'-CCATCACAGTGAACCGGTCTC-3' \\
hsa-miR-145-5p & Forward: 5'-ACGTCCAGTTTCCCAGGAATCC-3' \\
hsa-miR-211-5p & Forward: 5'-CCTTCCCTTTGTCATCCTTCGC-3' \\
hsa-miR-144-3p & Forward: 5'-TACAGTATAGATGATGTAC-3' \\
hsa-miR-122a & Forward: 5'-TGGAGTGTGACAATGGTGTTTG-3' \\
U6 & Forward: 5'-CGCTTCGGCAGCACATATAC-3' \\
\hline
\end{tabular}

B

miRNA expession in bone tissue

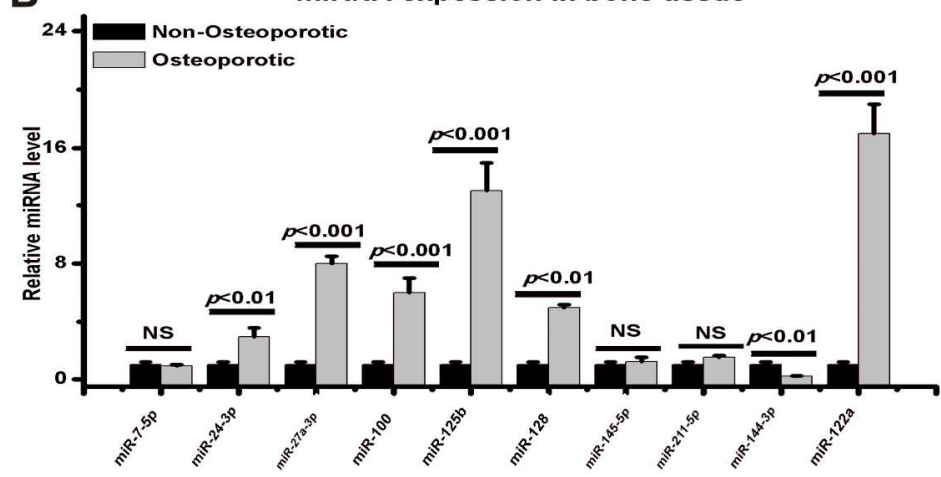

C

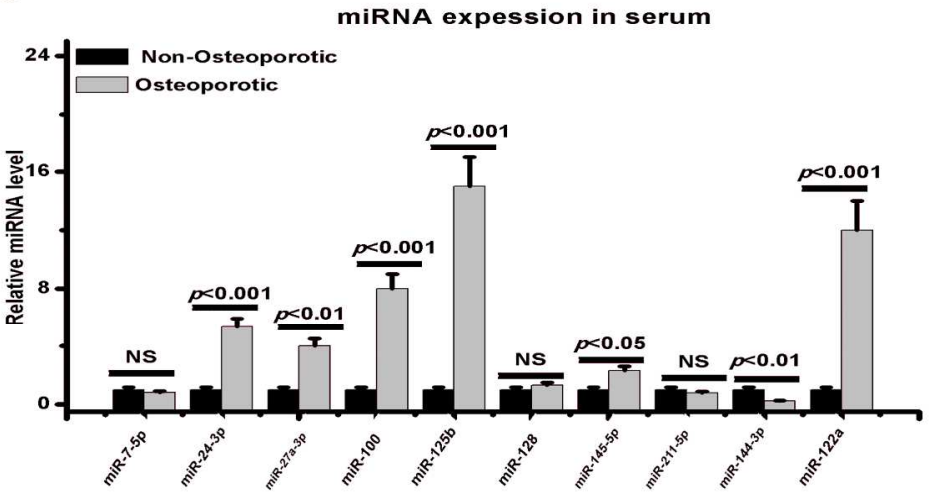

Fig. 1 miRNA expression patterns in bone and serum samples from Non-osteoporosis and osteoporosis (A) Selected candidate miRNAs, and corresponding qRT-PCR primer sequences. (B) Expression patterns of the selected candidate miRNAs in patient serum samples. (C) Expression patterns of the selected candidate miRNAs in patient bone samples. Expression levels are represented as the mean $\pm S . D$, with statistical significance being assessed via the Student's t-test $(p<0.05)$.

$146 \times 233 \mathrm{~mm}(300 \times 300$ DPI $)$ 

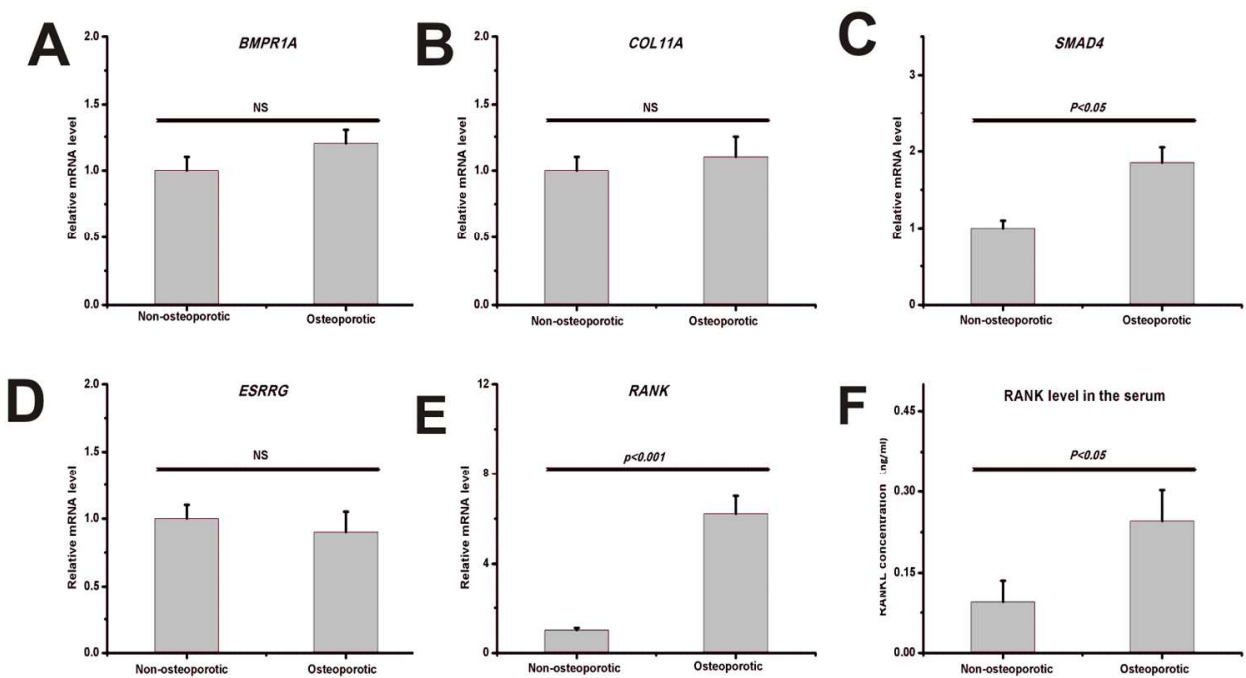

Fig. 2 Potential miR-144-3p target genes expression patterns in non-osteoporosis and osteoporosis. Online software was used to predict miR-144-3p target genes, and the resultant list of candidates was refined to include only those genes associated with bone homeostasis. Selected candidate genes were verified to be differentially expressed between non-osteoporosis and osteoporosis, via qRT-PCR. (A-E) The expression patterns of (A) BMPR1A, (B) COL11A, (C) SMAD4, (D) ESRRG, and (E) RANK in non-osteoporosis and osteoporosis in bone tissue. (F) The level of RANK production in patient serum samples was assessed via ELISA. Expression levels are represented as the mean \pm S.D., with statistical significance being assessed via the Student's t-test $(p<0.05)$.

$139 \times 77 \mathrm{~mm}(300 \times 300$ DPI $)$ 

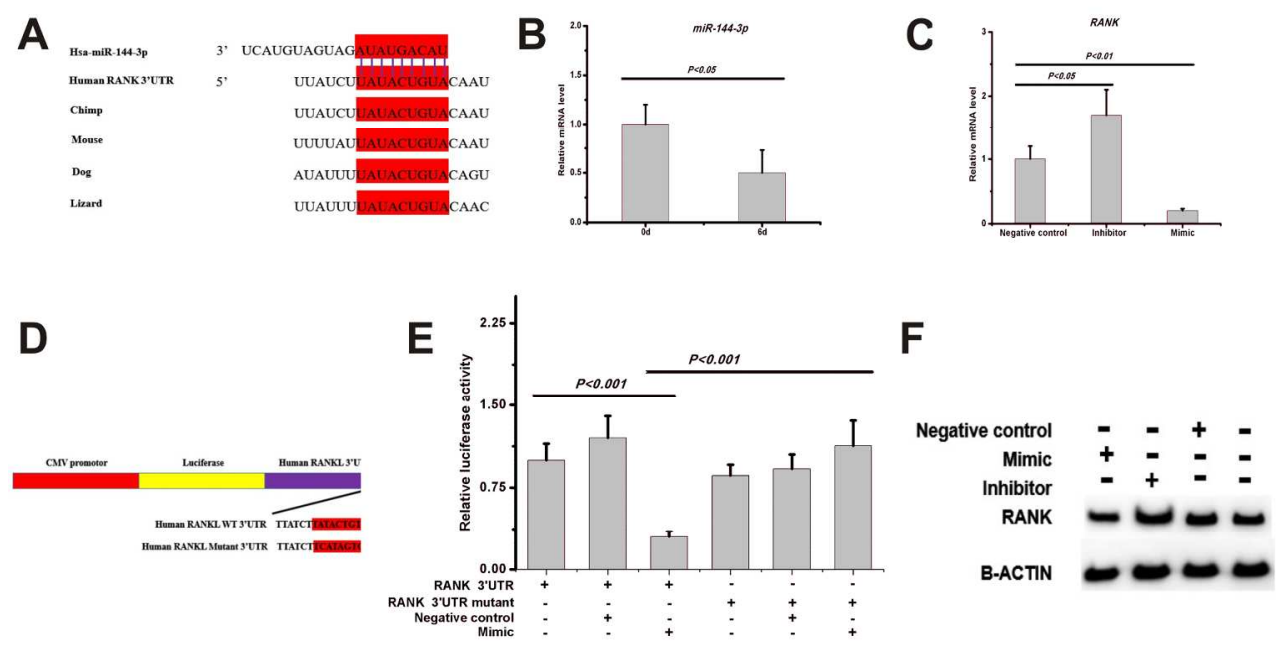

Fig. 3 RANK is a miR-144-3p target gene (A) Using TargetScans online software, we identified a miR-144-3p target site (red colour) in the RANK 3'UTR that is conserved in humans, chimps, mice, dogs, and lizards. (B) The expression of miR-144-3p was measured in CD14+ PBMCs using qRT-PCR, six days after the cells were induced to differentiate. (C) CD14+ PBMCs were transfected with the miR-44-3p mimic or inhibitor, and their expression of RANKL was then assessed via qRT-PCR. (D) A WT and mutant RANKL 3'UTR was cloned into the pMir-reporter vector to generate the CMV-luciferase-RANKL and CMV-luciferase-RANKL mutant 3'UTR vectors. (E) A cell-transfection experiment was performed to demonstrate that miR-144-3p inhibits RANKL expression in vivo. +, added; -, not added. (F) Western blot analysis demonstrating that miR-144-3p affects RANKL protein levels in vivo. + , added; - , not added. Data represent the mean \pm S.D., with statistical significance being assessed by the Student's t-test $(p<0.05)$.

$190 \times 98 \mathrm{~mm}(300 \times 300 \mathrm{DPI})$ 
A
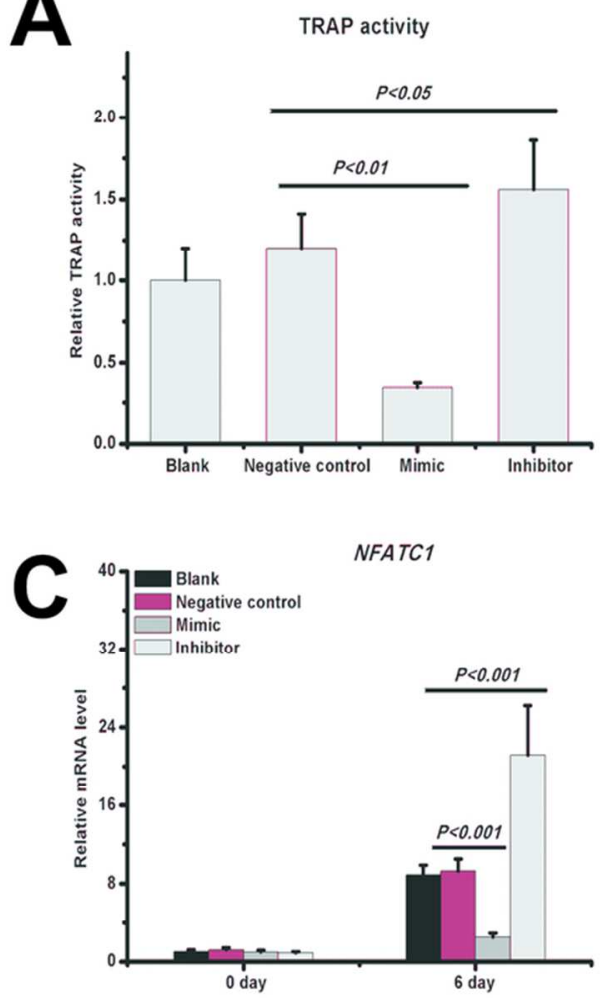

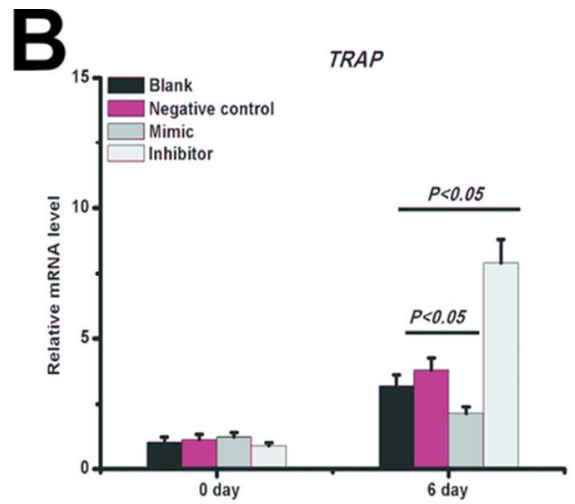

CTSK

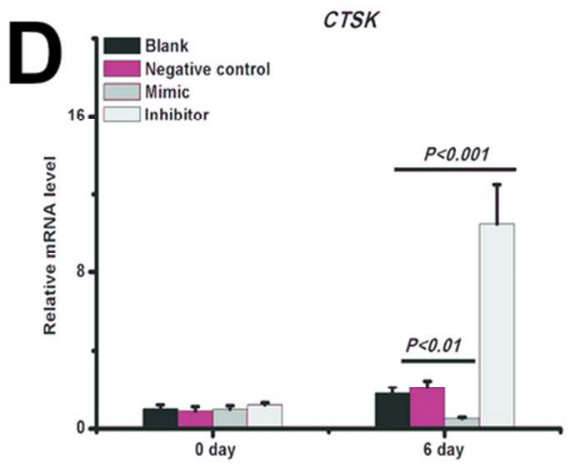

Fig. 4 miR-144-3p regulates the induction of CD14+ PBMCs to undergo osteoclastogenesis via M-CSF and RANKL treatment (A) CD14+ PBMCs were induced to differentiate via M-CSF and RANKL treatment and cultured for six days, before they exhibited TRAP activity. Transfection of the miR-144-3p mimic and inhibitor was shown to decrease and increase CD14+ PBMC TRAP activity, respectively. (B-D) qRT-PCR analysis of (B) TRAP, (C) NFATC, and (D) CTSK mRNA expression in response to transfection of CD14+ PBMCs with the miRNA-144-3p negative control, mimic, or inhibitor. Data represent the mean \pm S.D., with statistical significance being assessed by the Student's t-test $(p<0.05)$.

$78 \times 64 \mathrm{~mm}(300 \times 300 \mathrm{DPI})$ 


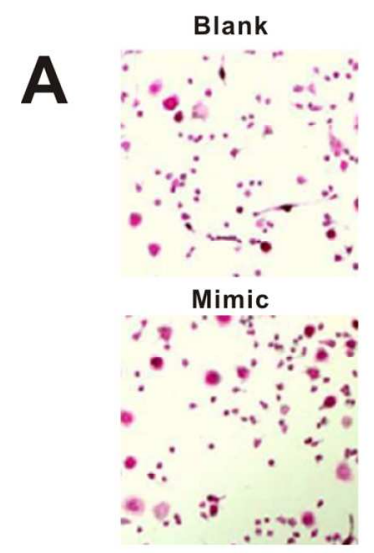

Negative control
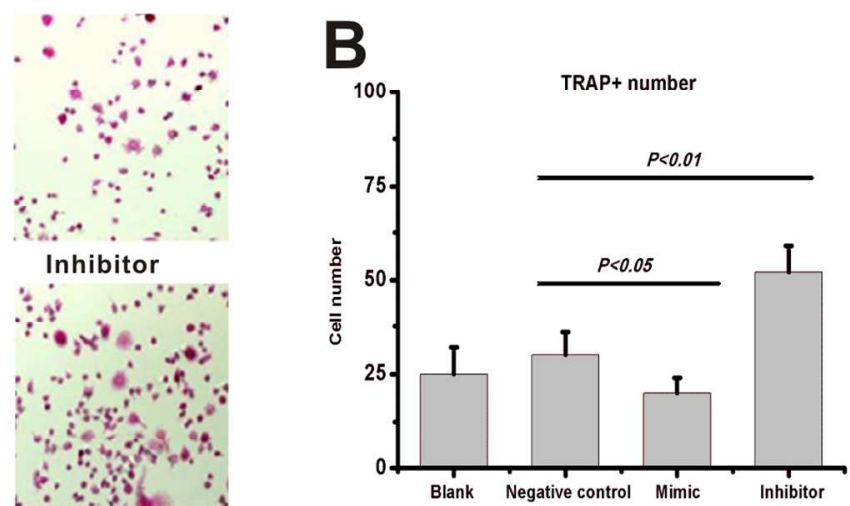

Fig. 5 miR-144-3p regulates osteoclast formation following the M-CSF and RANKL treatment of CD14+ PBMCs. (A) The cells were induced by the addition of M-CSF+RANKL for 6 days, after which the miR-144-3p mimic and inhibitor was added. (B) The statistics of the number of TRAP-positive cells. Statistical significance was assessed using the Student's t-test $(p<0.05)$.

$$
125 \times 56 \mathrm{~mm}(300 \times 300 \text { DPI })
$$




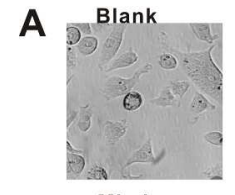

Mimic

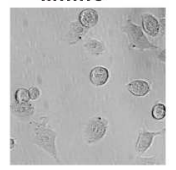

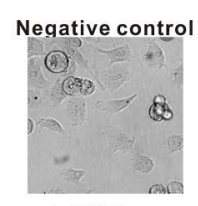

Inhibitor

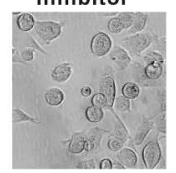

B

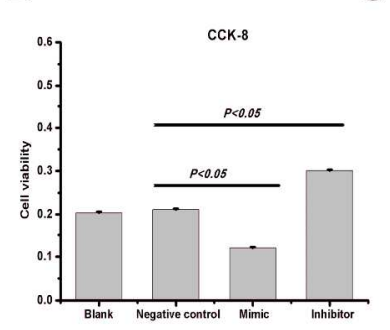

C

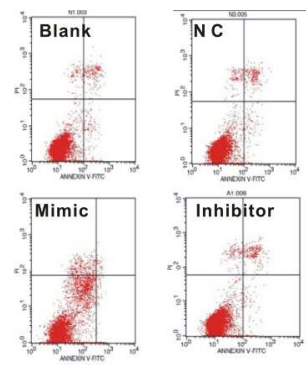

Fig. 6 Effects of transfecting CD14+ PBMCs with the miR-144-3p mimic, inhibitor, or negative control on cell proliferation and apoptosis (A) A CCK-8 assay was performed to evaluate the effect of transfecting CD14+ PBMCs with the miR-144-3p mimic, inhibitor, and/or negative control on cell proliferation. (B) A statistical analysis of the results revealed that the miR-144-3p mimic and inhibitor inhibited and increased CD14+ PBMC proliferation, respectively. (C) The effects of miR-144-3p mimic, inhibitor, and/or negative control transfection on CD14+ PBMC apoptosis was analysed via flow cytometry. Data represent the mean \pm S.D., with statistical significance being assessed by the Student's t-test $(p<0.05)$.

$220 \times 66 \mathrm{~mm}(300 \times 300 \mathrm{DPI})$ 